\title{
Disordered photonic crystals understood by a perturbation formalism
}

\author{
Zhi-Yuan Li, Xiangdong Zhang, and Zhao-Qing Zhang \\ Department of Physics, The Hong Kong University of Science and Technology, Clear Water Bay, Kowloon, Hong Kong, China
}

(Received 6 July 1999; revised manuscript received 11 November 1999)

\begin{abstract}
Photonic band gaps in disordered two-dimensional photonic crystals are investigated for two typical types of randomness: cylinder site displacements (site randomness) and cylinder radius variations (size randomness). The plane-wave expansion method with a supercell technique is applied to calculate the density-of-states (DOS) for the disordered photonic crystals. In particular, numerical simulations on the DOS for square and triangular lattices of dielectric cylinders in air with the $E$-polarization mode show that photonic band gaps are far more sensitive to disorders with a size randomness than with a site randomness. The first and second band gaps both reduce very little even for a site randomness of a strength as large as half the cylinder radius, yet they reduce more than one-half for a size randomness of a strength about one-third the cylinder radius. This substantial contrast can be understood by the analysis of the electromagnetic fields in disordered crystals. Based on such a field analysis, a perturbation formalism is proposed for disordered crystals and it accords well with the DOS calculations for a site randomness of even a moderate strength. At very weak size randomness, the perturbation method also works well to some extent. Such a simple perturbative analysis should provide a systematic way to understand various disordered photonic crystals qualitatively and even semiquantitatively.
\end{abstract}

\section{INTRODUCTION}

In recent years the fabrication of photonic crystals has attracted extensive interest. ${ }^{1,2}$ These artificial periodical structures exhibit "forbidden' frequency region where electromagnetic (EM) waves cannot propagate for both polarizations along any directions. This may bring about some peculiar physical phenomena, ${ }^{3-6}$ such as the suppression of the spontaneous emission of molecules and the localization of EM waves. Besides the academic interest, these structures imply the possible application in several scientific and technical areas such as filters, optical switches, cavities, design of low-threshold lasers, and high-efficient light-emitting diodes. $^{1,2}$ It is also proposed that such photonic crystals may hold the key to the continued progress towards all-optical integrated circuits. ${ }^{7}$

The fabrication of photonic crystals requires state-of-theart lithography techniques, such as electron-beam lithography and x-ray lithography. Despite the fact that rapid progress has been made in such techniques and some demonstrations on three-dimensional (3D) photonic crystals with a micrometer size have been reported based on a layer-bylayer growth scheme, the fabrication of three-dimensional (3D) photonic crystals with a band gap in the visible or infrared regimes still remains a difficult and challenging task. ${ }^{8-11}$ In contrast, the fabrication of 2D photonic crystals is much easier by such lithography techniques in the micrometer or submicrometer size regimes. ${ }^{12-15}$ Another technique that has been extensively studied to grow a 3D photonic crystal in the optical frequency is through the selfarrangement of colloid. Many self-assembling photonic crystals exist, including colloidal systems ${ }^{16,17}$ and artificial opals. ${ }^{18-20}$ This is rather a chemical method. Very recently, it was reported that porous carbons that are threedimensionally periodic on the scale of optical wavelengths could be made by a synthesis route resembling the geological formation of natural opal. ${ }^{21}$ In a similar scheme, the successful preparation of 3D photonic crystals consisting of air sphere in titania $\left(\mathrm{TiO}_{2}\right)$ with radii between 120 and $1000 \mathrm{~nm}$ was reported, which provided a fascinating prospect of opening photonic band gaps in the optical frequency. ${ }^{22}$

Nonuniformities inevitably occur in the fabrication of photonic crystals, especially when the crystals are of micrometer and submicrometer sizes. Among them are deviation of crystal "atoms" ( cylinders or spheres) from their periodic lattice sites, fluctuation of "atom", radii, configuration nonuniformity of "atoms," and so on. These disorders may affect the properties of photonic crystals significantly. For example, if the disorder in crystals reduces the photonic band gap appreciably enough, then the superior optical characters will be destroyed completely. For this reason, in recent years, there have appeared extensive discussions on disordered photonic crystals both theoretically ${ }^{23-27}$ and experimentally. ${ }^{28}$ The theoretical efforts include the bandgap calculations via the plane-wave expansion method with a supercell technique and the transmission spectrum calculations. It was found that the ground band gap could survive to a rather large amount of disorder both in 2D and 3D crystals. However, these simulations require powerful numerical calculations. In addition, they are still short of a clear understanding in physics for such disordered photonic crystals.

In this paper we will propose a perturbation formalism to understand the disordered photonic crystals in a systematic way. It is based on the analysis of the electromagnetic fields in the crystal with use of the plane-wave expansion method combined with the supercell technique. In particular, we have considered two typical disorders in a 2D photonic crystal consisting of dielectric cylinders in air: One is the random displacement of cylinders from their lattice sites (site randomness), and the other is the random fluctuation of cylinder radii (size randomness). The effect of disorders on the ground and higher photonic band gaps is measured via the density-of-states (DOS) for a disordered crystal. We will make comparisons between the perturbative calculations and DOS manipulations, and then show the effectiveness of the proposed perturbation formalism for weak disorders.

This paper is arranged as follows. In Sec. II we first briefly introduce the model and formalism of the supercell 
technique and the perturbation method for disordered 2D photonic crystals. Then in Sec. III we investigate in detail the effect of the site and size randomness on photonic band gaps and make a comparison between them. In Sec. IV we will apply the perturbation formalism in disordered photonic crystals, and discuss its effectiveness compared with the DOS calculations by the supercell technique. Then in Sec. V, we will analyze the electromagnetic field in the disordered crystals in detail to understand the different behaviors of the two kinds of randomness on the band gap and confirm the perturbation method. Finally the summary and conclusions are made in Sec. VI.

\section{MODEL AND FORMALISM}

\section{A. Supercell method for disordered photonic crystals}

The disordered photonic crystals are well described by the random strength. For an original periodic crystal with a lattice constant of $a$ and a cylinder radius of $r_{0}$, in the site randomness, every cylinder keeps its radius as $r_{0}$, while the $x$ and $y$ components of the position of the $i$ th cylinder in the disordered crystal, differ from those of the periodic case by $\gamma_{x} a$ and $\gamma_{y} a$, respectively, where $\gamma_{x}$ and $\gamma_{y}$ are variables uniformly distributed over the interval of $\left[-d_{x y}, d_{x y}\right]$. Here $d_{x y}$ is the random strength of the disordered system. For the disordered crystal with a size randomness of strength $d_{r}$, the cylinders are arrayed in the original lattice sites, while the radius of the $i$ th cylinder is given by $r_{i}=r_{0}+\gamma_{r} a$ where $\gamma_{r}$ is a random variable uniformly distributed over the interval $\left[-d_{r}, d_{r}\right]$.

The electromagnetic problem in such 2D disordered crystals can be solved with the use of the plane-wave expansion method combined with a supercell technique. In this way, the Maxwell's equations can reduce to the wave equation for the magnetic field as

$$
\nabla \times\left[\frac{1}{\epsilon(\mathbf{r})} \nabla \times \mathbf{H}(\mathbf{r})\right]=\frac{\omega^{2}}{c^{2}} \mathbf{H}(\mathbf{r}),
$$

where $\epsilon(\mathbf{r})$ is the dielectric function of the random system, $\omega$ is the frequency, and $c$ is the speed of light in vacuum. When the random strength is zero, i.e., perfect crystals, the frequency and electromagnetic field distributions can be solved with use of the plane-wave expansion method. ${ }^{29-31}$ For a disordered crystal, the plane-wave expansion method is still applicable, provided that it is combined with the supercell technique where the disordered system is approximated as a periodic system with a period large enough to guarantee negligible coupling between neighboring supercells.

In our simulations for both square and triangular lattices of dielectric cylinders in air, we only consider the $E$-polarization mode where the electric field is parallel to the extension axis of cylinders. For the $H$-polarization mode, the band-gap is much narrower. ${ }^{2,30}$ In our calculations for disordered crystals, we employ a supercell containing $5 \times 5$ unit cells for systems with site randomness, and $7 \times 7$ unit cells for systems with size randomness. In the former case, we use 729 plane waves in calculations, while for the latter case, 961 plane waves are used. In order to check the accuracy of our calculations, we first consider a periodic structure, where the band structures can be accurately determined using a single unit cell. We find the band-gap size obtained from a supercell calculation agrees with that obtained from a single unit cell to within $1 \%$.

The size of photonic band gaps in disordered crystals is determined by their DOS, which are obtained by solving Eq. (1) at each of over 10000 uniformly spaced values of Bloch's wave vector inside the first Brillouin zone of a unit cell. We have also changed the configuration of disordered crystals, adopting different random number series. It is found that the calculated DOS and band-gap size are insensitive to the sequences of random numbers used in the case of site randomness, even at large random strength. However, the results are quite dependent on the supercell configurations for the size randomness, especially at strong disorders. Therefore, the calculation results shown in this paper are all obtained by averaging five supercell configurations.

\section{B. Perturbation method for disordered photonic crystals}

Calculations of DOS with use of the plane-wave expansion method combined with a supercell technique requires powerful numerical computations. So it is of great interest to find a simple method that is qualitatively correct and approximately accurate in quantity. With this in mind, in the following we will extend the perturbation theory that is widely applied in quantum mechanics to the electromagnetism. One can find the perturbation formalism in a standard textbook of quantum mechanics. Following the basic concept therein, we will show how an eigenstate of a perfect crystal is affected by the introduction of disorders, which can be assumed as a perturbation when the disorder strength is weak. In fact, recently such a perturbation formalism has been successfully evaluated to engineer photonic crystals with large absolute band gaps. ${ }^{32}$

Consider a Bloch's state with an eigenfrequency of $\omega_{0}(\mathbf{k})$ and an eigenfield of $\mathbf{H}_{0}(r)$. They satisfy Eq. (1) as

$$
\nabla \times\left[\frac{1}{\epsilon_{0}(\mathbf{r})} \nabla \times \mathbf{H}_{0}(\mathbf{r})\right]=\frac{\omega_{0}^{2}}{c^{2}} \mathbf{H}_{0}(\mathbf{r}),
$$

where $\epsilon_{0}(\mathbf{r})$ is the periodic dielectric function of the perfect crystal. The eigenvalue can be written in an integral form as

$$
\begin{aligned}
\frac{\omega_{0}^{2}}{c^{2}} \int_{V}\left|\mathbf{H}_{0}(\mathbf{r})\right|^{2} d \mathbf{r} & =\int_{V} \nabla \times\left[\frac{1}{\epsilon_{0}(\mathbf{r})} \nabla \times \mathbf{H}_{0}(\mathbf{r})\right] \cdot \mathbf{H}_{0}^{*}(\mathbf{r}) d \mathbf{r} \\
& =\int_{V} \frac{1}{\epsilon_{0}(\mathbf{r})}\left|\nabla \times \mathbf{H}_{0}(\mathbf{r})\right|^{2} d \mathbf{r},
\end{aligned}
$$

where $V$ is the volume of crystal. In deriving the final formalism, we have considered the Maxwell's equations.

Then, for a disordered crystal with dielectric function $\epsilon(\mathbf{r})$ which can be regarded as a perturbation to $\epsilon_{0}(\mathbf{r})$, similarly we have

$$
\frac{\omega^{2}}{c^{2}} \int_{V}|\mathbf{H}(\mathbf{r})|^{2} d \mathbf{r}=\int_{V} \frac{1}{\epsilon(\mathbf{r})}|\nabla \times \mathbf{H}(\mathbf{r})|^{2} d \mathbf{r} .
$$

In the first-order perturbation formalism, the wave functions are kept as the unperturbed ones, so Eq. (4) now reads 

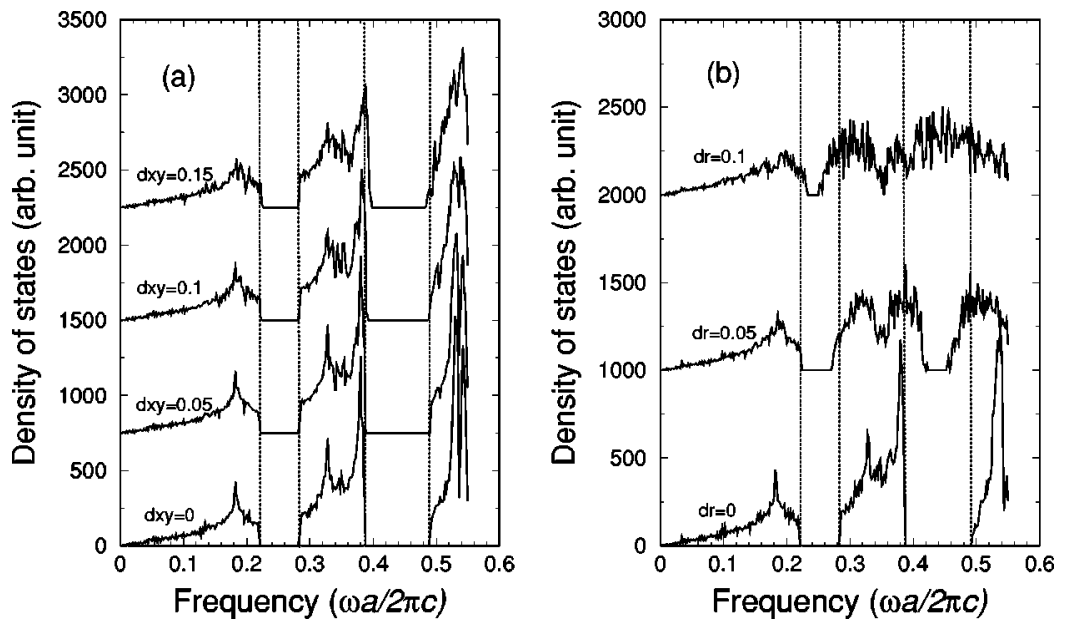

FIG. 1. Density of states (DOS) of disordered crystals with (a) site randomness and (b) size randomness in various random strengths. The DOS is calculated for the E-polarization mode of a square lattice of dielectric cylinders in air. The cylinders have a filling fraction of $f=0.3$ and a dielectric constant of $\epsilon=12.96$. Dotted vertical lines in (a) and (b) represent the band edges of a periodic crystal. For a clarity of view, the upper curves are offset alternately.

$$
\frac{\omega^{2}}{c^{2}} \int_{V}\left|\mathbf{H}_{0}(\mathbf{r})\right|^{2} d \mathbf{r} \cong \int_{V} \frac{1}{\epsilon(\mathbf{r})}\left|\nabla \times \mathbf{H}_{0}(\mathbf{r})\right|^{2} d \mathbf{r} .
$$

Combining it with Eq. (3), we have

$$
\begin{aligned}
\frac{\omega^{2}}{\omega_{0}^{2}}-1 \cong \frac{\int_{V}\left[\epsilon^{-1}(\mathbf{r})-\epsilon_{0}^{-1}(\mathbf{r})\right]\left|\nabla \times \mathbf{H}_{0}(\mathbf{r})\right|^{2} d \mathbf{r}}{\int_{V} \epsilon_{0}^{-1}(\mathbf{r})\left|\nabla \times \mathbf{H}_{0}(\mathbf{r})\right|^{2} d \mathbf{r}} \\
=\frac{\int_{V}\left[\epsilon^{-1}(\mathbf{r})-\epsilon_{0}^{-1}(\mathbf{r})\right]\left|\mathbf{D}_{0}(\mathbf{r})\right|^{2} d \mathbf{r}}{\int_{V} \epsilon_{0}^{-1}(\mathbf{r})\left|\mathbf{D}_{0}(\mathbf{r})\right|^{2} d \mathbf{r}},
\end{aligned}
$$

where $\mathbf{D}_{0}$ is the electric displacement field in the perfect crystal.

The shift of photonic band by disorders in crystals can be estimated by this perturbation formalism. It is a general form for various photonic crystals. For a 2D photonic crystal, which is excited by an $E$-polarization mode, we can derive another perturbation formalism regarding the electric field $\mathbf{E}(r)$ as $^{32}$

$$
\frac{\omega^{2}}{\omega_{0}^{2}}-1 \cong \frac{\int_{S}\left[\epsilon_{0}(\mathbf{r})-\epsilon(\mathbf{r})\right]\left|\mathbf{E}_{0}(\mathbf{r})\right|^{2} d \mathbf{r}}{\int_{S} \epsilon_{0}(\mathbf{r})\left|\mathbf{E}_{0}(\mathbf{r})\right|^{2} d \mathbf{r}},
$$

where $S$ is the area of the $2 \mathrm{D}$ crystal. Basically Eq. (7) is equivalent to Eq. (6) in the manner of the first-order perturbation. For instance, when $\epsilon_{0}(\mathbf{r})$ increases by a small value to $\epsilon(\mathbf{r})$, the eigenfrequency should decrease from the physical viewpoint. Indeed, Eqs. (6) and (7) both verify such an expectation. However, as $\mathbf{E}(r)$ is continuous everywhere inside the $2 \mathrm{D}$ crystal at the $E$-polarization mode, it is more accurate in numerical calculations for band shift to use Eq. (7) with $\mathbf{E}(r)$ than to use Eq. (6) with $\mathbf{H}(r)$.

It should be noted that the perturbation formalism introduced here is obtained by rather a simple physical consideration. Nevertheless, it may provide a reasonable estimate of band gaps in weak disordered photonic crystals, where the electromagnetic fields do not vary much compared with those in the perfect crystals. More accurately, one should concern the perturbation of systems with continuous spectra, and with many degenerate states, which is common in photonic crystals. However, such a rigorous treatment will require extensive numerical computations, which might be even more time consuming than the supercell method.

\section{CALCULATION RESULTS OF DOS FOR SITE AND SIZE RANDOMNESS}

In our calculations, the dielectric constant of cylinders is fixed as $\epsilon=12.96$. We first consider a square lattice of such cylinders in air with a filling fraction of $f=0.3$. The corresponding radius of cylinders is $r_{0}=0.309 a$, where $a$ is the lattice constant of the crystal. In a perfect crystal, two large band gaps open at $0.219-0.284(2 \pi c / a)$ and 0.385 $-0.490(2 \pi c / a)$, respectively. The normalized band-gap width $\Delta \omega / \omega_{g}$ for these two gaps are $25.8 \%$ and $24.0 \%$. Here $\Delta \omega$ and $\omega_{g}$ denote the width and the center frequency of the band gap, respectively. It may be expected that such large band gaps could survive to a rather large amount of disorder in crystals.

To measure the effect of various disorders on the band gap, we calculate the DOS of the corresponding disordered crystal with a supercell technique. The results are displayed in Figs. 1(a) and 1(b) for various strengths of site randomness and size randomness, respectively. For a clarity of view, the upper curves in both panels are offset alternately from the bottom curve. It is evident that the band gaps are much more sensitive to the fluctuation of cylinder radii than the displacement of cylinder sites. The ground band gap lies at 0.220 $-0.284(2 \pi c / a), \quad 0.221-0.284(2 \pi c / a)$, and 0.224 $-0.283(2 \pi c / a)$ for site randomness with a strength of $d_{x y}$ $=0.05,0.10$, and 0.15 , respectively. The corresponding normalized band-gap widths are $25.4 \%, 25.0 \%$, and $23.2 \%$. So at weak disorder, the reduction of the ground band gap is negligibly small. Even for a strong disorder with a strength as large as half the cylinder radius, the band gap only reduces about $10 \%$. In contrast, for size randomness with a strength of $d_{r}=0.05$ and 0.10 , the ground band gap reduces to $0.222-0.272(2 \pi c / a)$ and $0.232-0.252(2 \pi c / a)$ with a normalized width of $20.2 \%$ and $8.3 \%$, respectively. The band gap reduces almost to one-third for a moderate disorder strength of about one-third of the cylinder radius. 

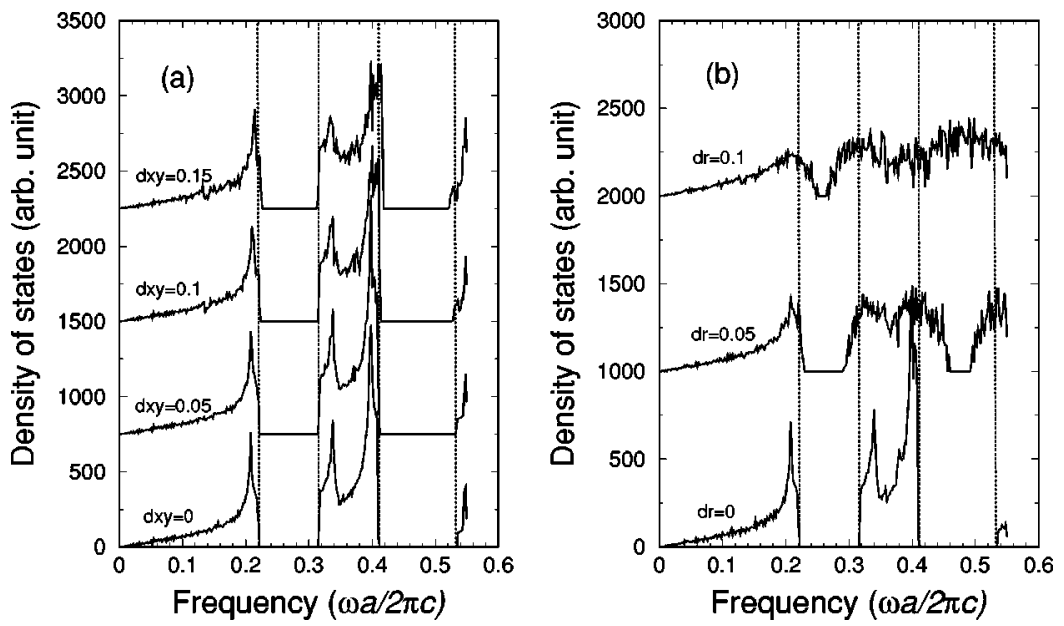

FIG. 2. Density of states (DOS) of disordered crystals with (a) site randomness and (b) size randomness in various random strengths. The DOS is calculated for the E-polarization mode of a triangular lattice of dielectric cylinders in air with $f=0.3$ and $\epsilon=12.96$. The upper curves are also offset alternately.

The contrast is even more appreciable for the higher band gap. For a disordered crystal with site randomness of strength of $d_{x y}=0.05,0.10$, and 0.15 , the second band gap reduces to $0.386-0.490(2 \pi c / a), 0.389-0.488(2 \pi c / a)$, and $0.395-0.484(2 \pi c / a)$ with a normalized width of $23.7 \%, 22.6 \%$, and $22.2 \%$, respectively. The reduction of the band gap is less than $20 \%$ for a site disorder with a strength of half the cylinder radius. Therefore, this higher band gap can also endure a rather large displacement of cylinders from their lattice sites. As a contrast, for a size randomness of $d_{r}=0.05$, the second band gap reduces to $0.422-0.454(2 \pi c / a)$, and the normalized width $(7.3 \%)$ is reduced more than one-half that of the perfect crystal. At a stronger disorder of $d_{r}=0.10$, this higher band gap is closed. More detailed calculations show that it vanishes at about $d_{r}$ $=0.065$.

Numerical simulations for triangular lattices of dielectric cylinders in air show similar characters. Figures 2(a) and 2(b) demonstrate the DOS of disordered crystals of $f=0.3$ and $r_{0}=0.288 a$ with site and size randomness, respectively, in various random strengths. Here the upper curves of DOS are also offset alternately. For a perfect crystal, two band gaps open at $0.220-0.316(2 \pi c / a)$ and 0.409 $-0.530(2 \pi c / a)$. The normalized gap widths are $35.8 \%$ and $25.7 \%$, respectively. The first band gap is reduced to 0.220 $-0.316(2 \pi c / a), \quad 0.222-0.316(2 \pi c / a), \quad$ and 0.224 $-0.315(2 \pi c / a)$ for a disordered crystal with a site randomness of $d_{x y}=0.05,0.10$, and 0.15 , and to 0.227 $-0.293(2 \pi c / a)$ and $0.250-0.266(2 \pi c / a)$ for a size randomness of $d_{r}=0.05$ and 0.10 , respectively. The corresponding gap widths are $35.8 \%, 34.9 \%, 33.7 \%$, and $25.4 \%$, $6.2 \%$, respectively. It is evident that the band gap is far more sensitive to the fluctuation of cylinder radii. The second band gap has a reduction at the site randomness as small as the first band gap. It lies at $0.410-0.529(2 \pi c / a), 0.412$ $-0.526(2 \pi c / a)$, and $0.416-0.521(2 \pi c / a)$ for $d_{x y}$ $=0.05,0.10$, and 0.15 , respectively, with a width of $25.3 \%$, $24.3 \%$, and $22.4 \%$ correspondingly. However, the reduction of this higher band gap by a size randomness is far larger than that of the first band gap. At $d_{r}=0.05$, the band gap $[0.458-0.493(2 \pi c / a)]$ has a width about one-third that of the perfect crystal. It is closed at $d_{r} \approx 0.065$.

We have also investigated the disordered crystals in different filling fractions. The results are shown in Figs. 3 and 4 for square and triangular lattices of dielectric cylinders in air, respectively. In the plots, the edge frequency of the ground and the second band gaps for a disordered crystal with site and size randomness of the same strength fixed as $d_{x y}=d_{r}$ $=0.05$ are compared with those of the perfect crystal. It is evident that at various filling fractions, the reduction of both band gaps by a size fluctuation is more remarkable than that caused by a site displacement, especially for the second band gap. Furthermore, the reduction of band gaps by a size fluctuation grows rapidly when the filling fraction decreases, yet at a site randomness it is not sensitive to the filling fraction. Qualitatively, lowering the filling fraction means growing the relative random strength. As we have shown in Figs. 1 and 2, the band gap is far more sensitive to the strength variation of size randomness than of site randomness.

\section{PERTURBATIVE CALCULATIONS FOR DISORDERED PHOTONIC CRYSTALS}

In the above, we have investigated band gaps in disordered photonic crystals via calculations of DOS with use of the plane-wave expansion method combined with a supercell technique. This requires extensive computational efforts. In the following we will apply the perturbation formalism [Eq. (7)] to understand disordered photonic crystals. We will

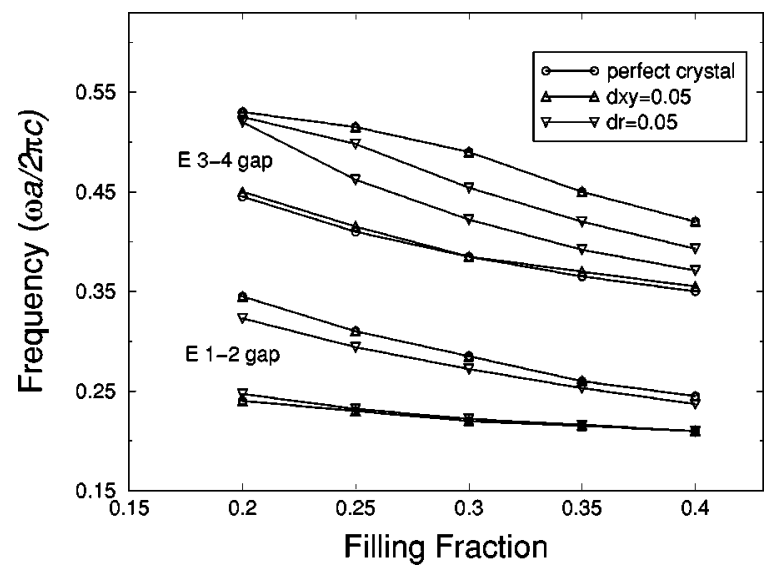

FIG. 3. Plots of edge frequencies of the $E$-polarization 1-2 and 3-4 band gaps for disordered square lattices in various filling fractions. The random systems with the site and size randomness are of the same strength as $d_{x y}=d_{r}=0.05$. 


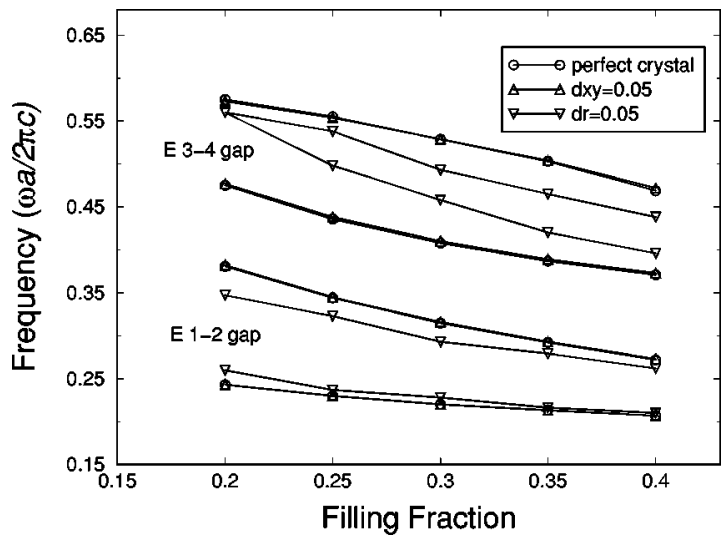

FIG. 4. Plots of edge frequences of the E-polarization 1-2 and 3-4 band gaps for disordered triangular lattices in various filling fractions for the site and size randomness of the same strength as $d_{x y}=d_{r}=0.05$.

compare it with the supercell technique and demonstrate its effectiveness.

To investigate the effect of disorders on band gaps, it is sufficient to consider only the edge states of the band gap, according to numerical simulations. We first solve photonic band structures of perfect crystals from Eq. (1) regarding the magnetic field $\mathbf{H}(r)$. The frequency $\omega_{0}$ and field distribution $\mathbf{H}_{0}(\mathbf{r})$ of the edge states can be obtained. Then we calculate the distribution of the electric field $\mathbf{E}_{0}(\mathbf{r})$ from Maxwell's equations. Applying Eq. (7) for the most probable configurations, the frequency shift of the edge states by disorders, and then the perturbative frequency, can be calculated.

Corresponding to Sec. III, we have considered the E-polarized 1-2 and 3-4 band gaps for a disordered square of dielectric cylinders in air. The result of edge frequencies calculated by the perturbation formalism is displayed in Fig. 5 for various random strengths of (a) site randomness, and (b) size randomness and for a filling fraction of $f=0.3$. As a comparison, we also plot the results calculated by the supercell method. According to the calculation of photonic band structures, the edge states of the first band gap for such a square lattice lie at high symmetry points of the Brillouin zone as $M 1$ and $X 2$, and for the second band gap they are at $X 3$ and $M 4$ points.

It is clear from Fig. 5(a) that the perturbation method works quite satisfactorily in the case of site randomness, compared with the supercell technique. Even at a large random strength like $d_{x y}=0.10$, which is one-third of the cylin- der radius, the error of perturbative calculation $\delta \omega / \omega$ is below $5.0 \%$. Here $\omega$ is the edge frequency, and $\delta \omega$ is the deviation of the calculated frequency by the perturbation method from that by the supercell method. The supercell method shows that the frequency shift of upper edge state is far smaller than the lower edge state for both the first and second band gaps. Indeed, this is also verified by the perturbation method. For instance, the frequency shift of the upper edge state at $d_{x y}=0.05$ obtained by the perturbation method is $-4 \times 10^{-4}(2 \pi c / a)$ and $-6 \times 10^{-4}(2 \pi c / a)$ for the first and second gaps, respectively, while for the lower edge state it is $1.6 \times 10^{-3}(2 \pi c / a)$ and $3.2 \times 10^{-3}(2 \pi c / a)$, respectively. According to the supercell method, such a frequency shift is $0 \times 10^{-3}(2 \pi c / a)$ for the upper edge states accurate to a value of $0.5 \times 10^{-3}(2 \pi c / a)$, while it is 1 $\times 10^{-3}(2 \pi c / a)$ and $1 \times 10^{-3}(2 \pi c / a)$, respectively for the lower edge states.

The accordance between the two methods is far less satisfactory in the case of size randomness, especially for the second band gap, as shown in Fig. 5(b). The width of the first band gap obtained by the perturbation approach is less than that by the supercell technique. For instance, the normalized width $\Delta \omega / \omega_{g}$ of the first band gap obtained by the perturbation method is $19.4 \%$ and $10.8 \%$ at $d_{r}=0.05$ and 0.10 , respectively. The error is $4 \%$ and $30 \%$, respectively, compared with those by the supercell technique of $20.2 \%$ and $8.3 \%$. The error of perturbative calculations are much larger for the higher band gap. At a random strength of $d_{r}=0.05$, the perturbative $\Delta \omega / \omega_{g}$ is $16.4 \%$ with an error of about $125 \%$ over the accurate one $(7.3 \%)$. The perturbative calculation shows the existence of a band gap even at $d_{r}=0.10$, while this band gap is closed at about $d_{r}=0.065$. However, at weak disorder strength, for example, $d_{r}=0.025$, the error is below $5 \%$. Therefore, the perturbation method can give rise to somewhat precise prediction of the ground band gap in disordered crystals with even a moderate size randomness.

The triangular lattice of dielectric cylinders in air demonstrates similar behavior to the square lattice. Figs. 6(a) and 6(b) display band frequencies calculated by the supercell and perturbation methods, respectively, for a disordered crystal of $f=0.3$ with various random strengths of (a) site randomness and (b) size randomness. In such a crystal, the edge states of the first and second band gap are at high-symmetry points of $J 1, X 2$, and $\Gamma 3, \Gamma 4$, alternately. For the ground band gap, at even moderate disorders (from 0.05 to 0.10 ) for both the site and size randomness, the perturbation method
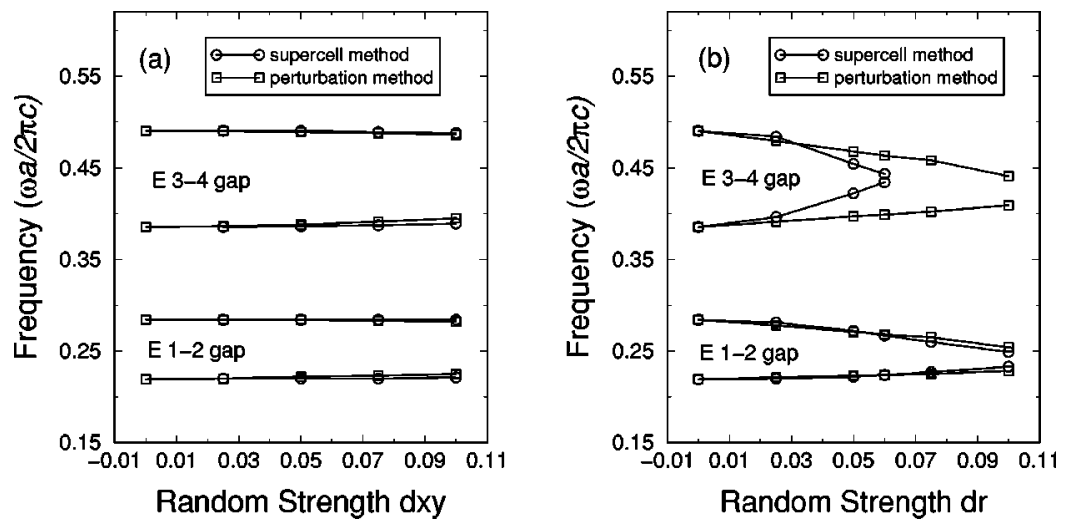

FIG. 5. Band-edge frequencies calculated by the supercell and perturbation methods, respectively, for a disordered square crystal with various random strengths for (a) site randomness and (b) size randomness. Other parameters are $f$ $=0.3$ and $\epsilon=12.96$. 

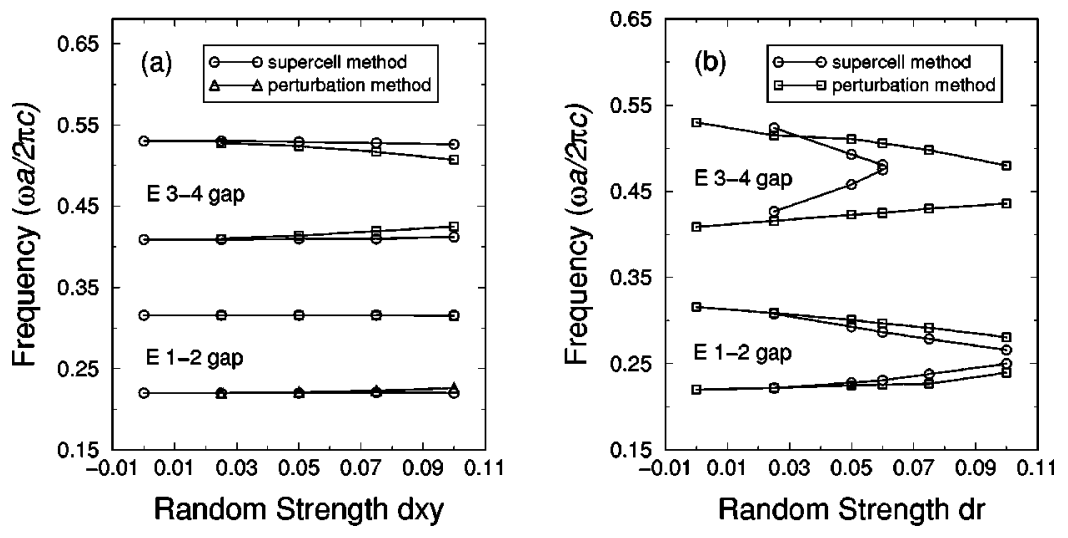

FIG. 6. Band-edge frequencies calculated by the supercell and perturbation methods, respectively, for a disordered triangular crystal with various random strengths for (a) site randomness and (b) size randomness. Other parameters are $f$ $=0.3$ and $\epsilon=12.96$. works quite well. The error of band gap width is generally below $10 \%$. Also similar to the square lattice, the error of the perturbative calculations for the higher band gap is very large for the size fluctuation of cylinders, while it is moderate for the site displacements.

\section{ANALYSES AND DISCUSSION ON ELECTROMAGNETIC FIELDS}

The above calculations by both the accurate supercell technique and an approximate perturbation formalism all demonstrate that photonic band gaps are far more sensitive to a fluctuation of cylinder radii than a displacement of cylinder sites from the perfect crystal. The calculations also show that a perturbation method based on the assumption that the electromagnetic fields in disordered crystals do not vary much compared with those in the perfect crystals can give rise to a somewhat precise prediction of the ground band gap. Therefore, it is necessary to investigate the electromagnetic fields in disordered crystals in order to find out the physical source of the different behaviors and then to confirm the effectiveness of the proposed perturbation method.
As mentioned above, the edge states of a perfect 2D square crystal with $f=0.3$ at the $E$-polarization mode lie at high-symmetry points of $M 1$ and $X 2$ for the first band gap, and $X 3$ and $M 4$ for the second band gap. We have calculated the electric field $\mathbf{E}(r)$ at these edge states with the use of the plane-wave expansion method. The amplitude distribution of $\mathbf{E}(r)$ is plotted in Fig. 7 at these edge states with Bloch waves of (a) M1: $(\pi / a)(1,1)$, (b) X2: $(\pi / a)(0,1)$, (c) X3: $(\pi / a)(0,1)$, and (d) M4: $(\pi / a)(1,1)$, respectively. Data are shown in $3 \times 3$ unit cells. For the $M 1$ state, the maximum of field lies at the center of the unit cell, while the field vanishes at the border of unit cell. The field at the $X 2$ state has two symmetric peaks at about the border of cylinder at the $(1,0)$ direction. Due to the symmetry of the crystal, the field is symmetric with respect to the $(0,1)$ line, where the field vanishes. The $X 3$ state is orthogonal to the $X 2$ state. The field shows two peaks inside the cylinder at the $(0,1)$ direction and vanishes along the $(1,0)$ direction, perpendicular to that at the $X 2$ state. The field at the M4 state shows four symmetric peaks at the $(1,1)$ and other three equivalent directions and the peaks are near the border of the cylinders. The field vanishes at both the $(0,1)$ and the $(1,0)$ directions. It is clear that
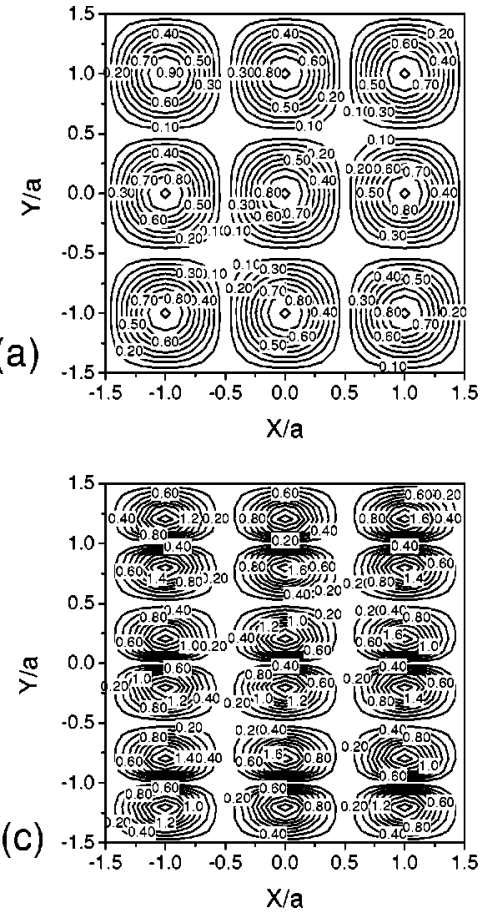

(b)
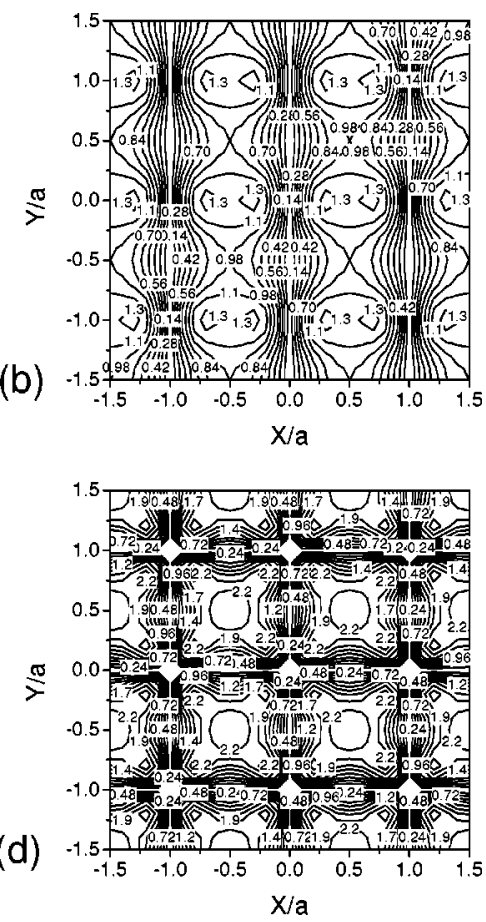

FIG. 7. Contour plot of the distribution of the electric-field amplitude in a perfect square crystal with $f=0.3$ and $\epsilon=12.96$ for the edge states corresponding to Bloch wave vectors of (a) M1: $(\pi / a)(1,1), \quad(\mathrm{b}) \quad \mathrm{X} 2: \quad(\pi / a)(0,1), \quad(\mathrm{c}) \mathrm{X} 3$ : $(\pi / a)(0,1)$, and (d) M4: $(\pi / a)(1,1)$. For a clarity of view, only data in $3 \times 3$ unit cells are shown. 

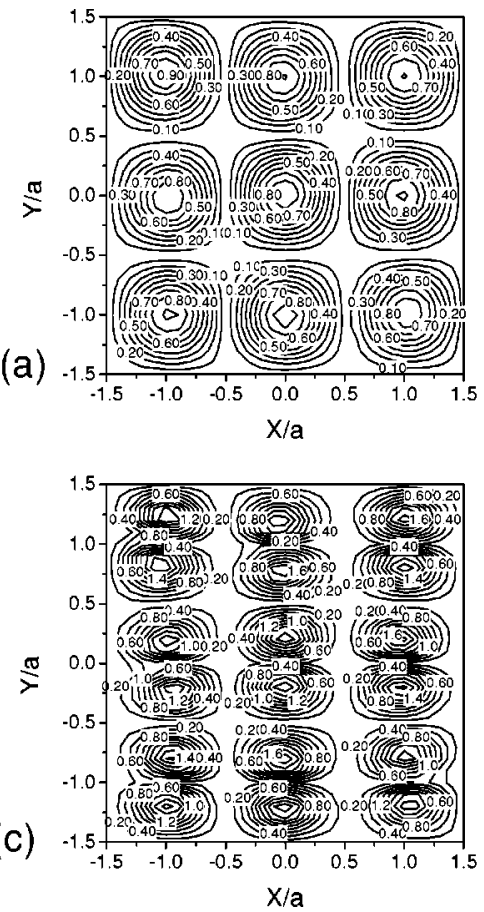
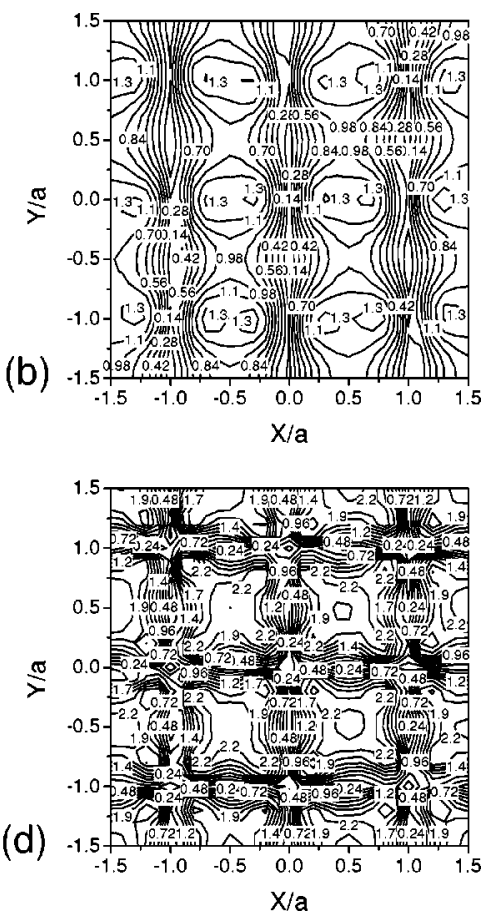

FIG. 8. Contour plot of the distribution of the electric-field amplitude in a disordered square crystal with a site randomness of strength $d_{x y}$ $=0.05$ for the edge states corresponding to Figs. $7(\mathrm{a})-7(\mathrm{~d})$ the field in these edge states demonstrates an exact symmetry.

The electric field in disordered crystals is calculated by the plane-wave expansion method with a supercell technique. Corresponding to Fig. 7, the amplitude of electric field in a disordered square crystal with a site randomness of various strengths such as $d_{x y}=0.05,0.10$, and 0.15 is displayed in Figs. 8, 9, and 10, respectively. In Fig. 8, data in $3 \times 3$ unit cells are plotted, while in Figs. 9 and 10, we display data in $5 \times 5$ unit cells. At a weak disorder of $d_{x y}=0.05$, as shown in Fig. 8, the field at all the four edge states has a weak redistribution, however, such a redistribution conforms exactly to the redistribution of cylinders, according to more detailed check of the configuration of the disordered crystal. Furthermore, the field magnitude changes even less, so the frequency has only a negligible shift, according to field-energy formalism of Eq. (4). When the random strength increases, the redistribution of the field will grow, in addition, the field magnitude might experience a remarkable change. This is indeed the case for a moderate disorder of $d_{x y}=0.10$, as shown in Fig. 9. Especially, the field magnitude in the $X 3$ edge state fluctuates remarkably in various cylinders. Furthermore, there appears an appreciable enhancement (about three times) of the field in several cylinders at the right side of Fig. 9(c). Therefore, it is expected that the frequency shift of the state will be far larger than other states. This assump-
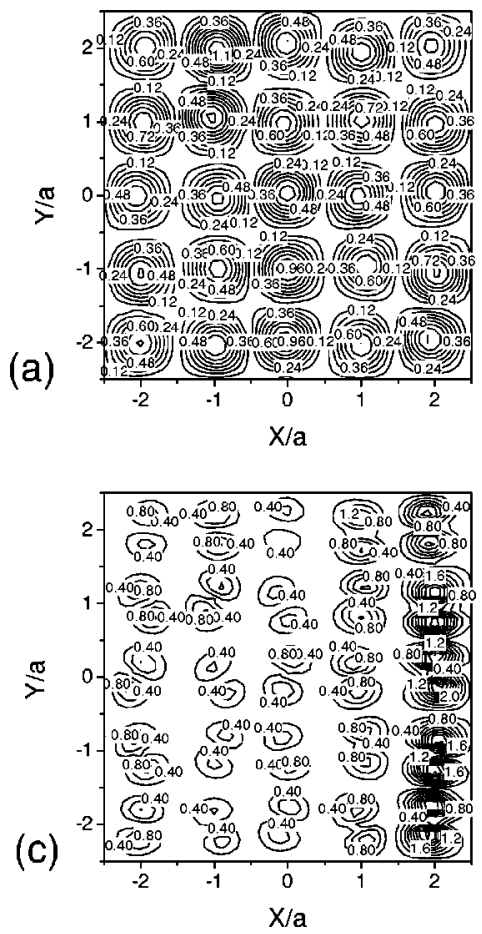
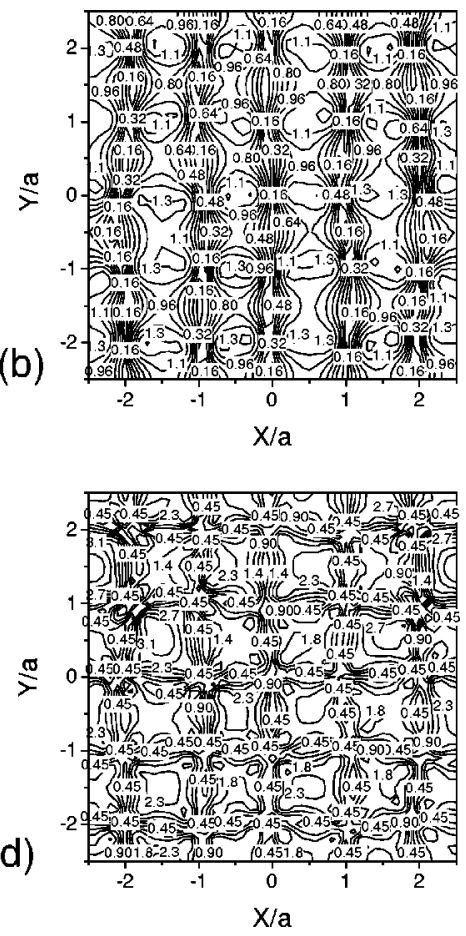

FIG. 9. The same as in Fig. 8, except for a disordered square crystal with a site randomness of strength $d_{x y}=0.10$. Data in $5 \times 5$ unit cells are plotted. 

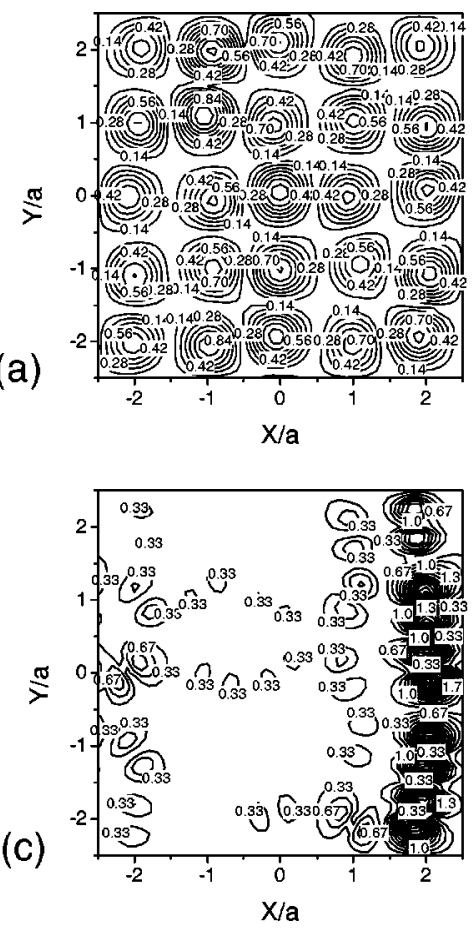
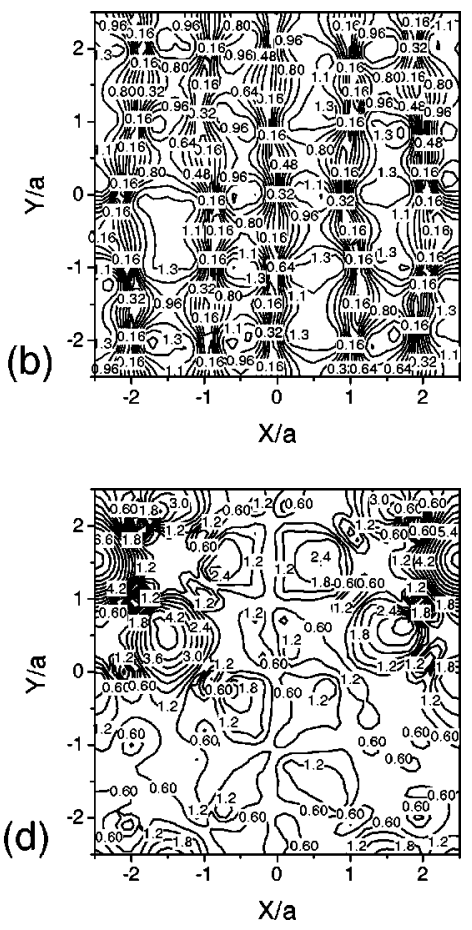

FIG. 10. The same as in Fig. 9, except for a disordered square crystal with a site randomness of strength $d_{x y}=0.15$. tion has been verified by the DOS calculations. It can also be seen that the field redistribution in the $X 2$ state is smaller than in the $M 1$ state as is the $M 4$ state compared to the $X 3$ state. So the frequency shift of the upper edge states is smaller than that of the lower edge states. All these characters have indeed been verified by the DOS calculations as well as the perturbative simulations.

As the disorder further grows to $d_{x y}=0.15$, the fluctuation and redistribution of the field in all the edge states becomes very appreciable. One can see that the field enhancement in the $X 3$ state becomes much stronger than in $d_{x y}=0.10$, and in other regions of the crystal, the field is greatly weakened. This signals the appearance of a localized state. There is also a localization sign of field in the $M 4$ state. Although the field varies much, the frequency shift caused by such a strong disorder is not very large, as shown in Fig. 1(a). It seems that the site randomness only results in a "shallow defect state" in the disordered crystal. This can be understood as follows. According to Eq. (4), the eigenfrequency of the disordered crystal is not determined by the field distribution of individual cylinders, but rather by an integration of all the cylinders. As it is an average magnitude, the frequency of the edge states does not shift much by random displacement of cylinders, even if the field fluctuation is very strong.

The situations are extremely different for disordered crystals with a size randomness. As noted in Sec. III in Figs. 1(b) and 2(b), the photonic band gap is sensitive to a radius fluctuation of cylinders. At a moderate disorder strength, the reduction of band gap is appreciable, especially for the higher band gap. In fact, one can find out such a character from the electric-field distribution inside a disordered crystal, which can be calculated by a supercell technique. In Figs. 11(a)-11(d), we display the field magnitude of a disordered crystal with a size randomness of $d_{r}=0.05$ corresponding to Fig. 7 for the four edge states, respectively. Data are plotted in $5 \times 5$ unit cells. There is a clear fluctuation and redistribution of fields at the $M 1$ and $X 2$ states, however no evident localization occurs. Checking out the configuration of cylinders, we find that the field in the $M 1$ state is enhanced at cylinders with smaller radii, and it is weakened at cylinders with larger sizes. For the $X 2$ state, the field distribution shows an inverse character. Comparing them with Figs. 7(a) and 7(b) for a perfect crystal, the frequency shift will be small due to the average over a large number of cylinders.

For the edge states of the higher band gap, the localization of field is very significant, as displayed in Figs. 11(c) and $11(\mathrm{~d})$. There appears at the $X 3$ state a very strong field peak localized around the cylinder at the coordinate $(2 a, a)$, and another slightly weaker peak around the cylinder at $(-2 a$, $-a)$. For the M4 state, two peaks appear around the cylinders lying at $(-a, a)$ (very strong) and at $(2 a, 0)$ (much weaker). The localization effect of field caused by a weak size randomness is much more remarkable than that caused by even a strong site randomness. This can be clearly seen by comparing Figs. 11(c) and 11(d) with Figs. 10(c) and 10(d). These edge states show the essential feature of "deep defect states." Therefore, the frequency shift in the former case is much larger, which results in a large (more than one-half) reduction of the band gap.

The localization character in the edge states of the first band gap becomes significant as the disorder strength of size randomness grows to a moderate value. In Figs. 12(a) and 12(b), we display the amplitude distribution of the electric field in the $M 1$ and $X 2$ states for a disordered crystal the same as in Fig. 11, except that now the random strength is $d_{r}=0.10$. The $X 3$ and $M 4$ states are not considered, as the 

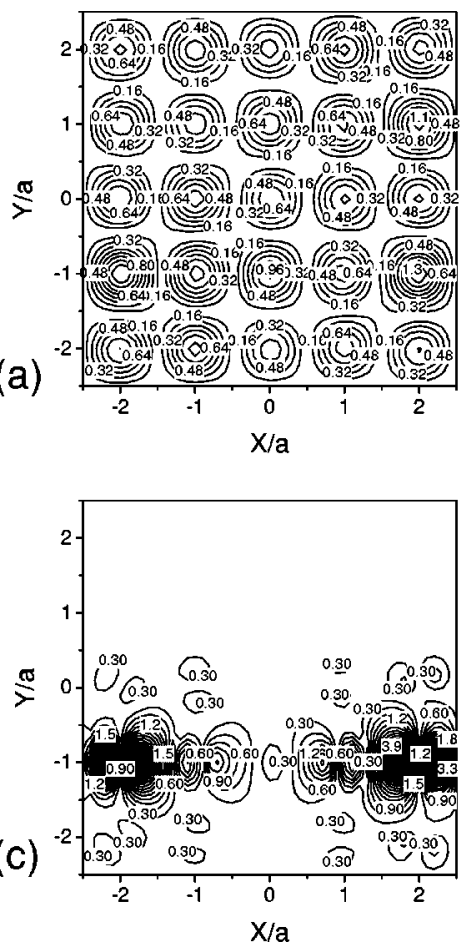

(b)
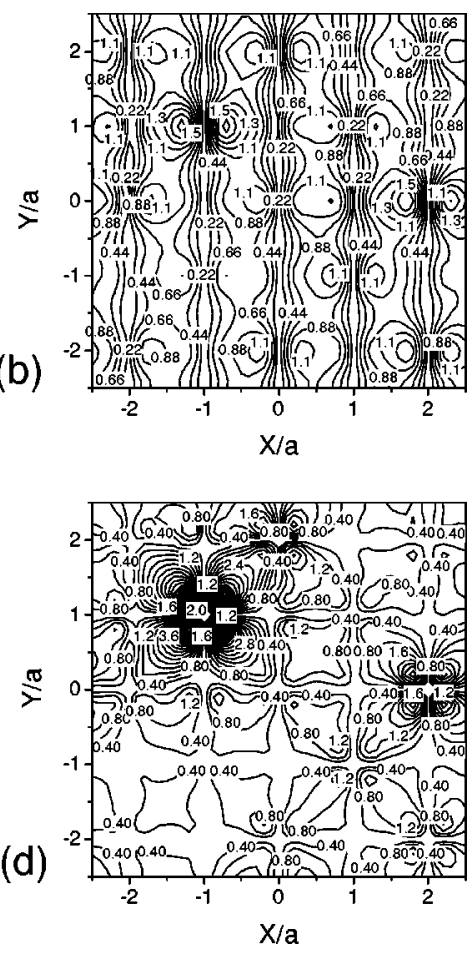

FIG. 11. Contour plot of the distribution of the electric-field amplitude in a disordered square crystal with a size randomness of strength $d_{r}$ $=0.05$ for the edge states corresponding to Figs. $7(\mathrm{a})-7(\mathrm{~d})$, respectively. Data are displayed in 5 $\times 5$ unit cells. band gap has been closed. Compared with Figs. 11(a) and 11(b), the localization of field grows quickly at the same regions as in the weak disorder, i.e., at $(-2 a,-a)$ and $(2 a,-a)$ in the $M 1$ state, and at $(-a, a)$ and $(2 a, 0)$ in the $X 2$ state. For such strongly localized edge states, the frequency shift is quite large, therefore, the band gap is greatly reduced.

The above analysis of electromagnetic fields in disordered photonic crystals ensures that the perturbation formalism proposed before can work well for disordered crystals with a site randomness with even a moderate strength. In contrast, this approximate method is less satisfactory for disordered crystal with even a weak size randomness, especially for the higher band gap. Remember that the perturbation method is essentially based on the approximate assumption that the electromagnetic fields in a disordered crystal do not vary much from those in a perfect crystal, when the disorder is weak.

In fact, the different behaviors of band-gap reduction by the site and size randomness can be interpreted by a simple consideration qualitatively based on the perturbation formalism of Eq. (7), where the field remains unchanged after in- troduction of disorders. When a cylinder displaces from its lattice site, the frequency shift is

$$
\begin{aligned}
\frac{\omega^{2}}{\omega_{0}^{2}}-1 \cong \frac{\int_{S_{1}}[\epsilon-1]\left|\mathbf{E}_{0}(\mathbf{r})\right|^{2} d \mathbf{r}+\int_{S_{2}}[-\epsilon+1]\left|\mathbf{E}_{0}(\mathbf{r})\right|^{2} d \mathbf{r}}{\int_{S} \epsilon_{0}(\mathbf{r})\left|\mathbf{E}_{0}(\mathbf{r})\right|^{2} d \mathbf{r}} \\
=\frac{[\epsilon-1]\left[\int_{S_{1}}\left|\mathbf{E}_{0}(\mathbf{r})\right|^{2} d \mathbf{r}-\int_{S_{2}}\left|\mathbf{E}_{0}(\mathbf{r})\right|^{2} d \mathbf{r}\right]}{\int_{S} \epsilon_{0}(\mathbf{r})\left|\mathbf{E}_{0}(\mathbf{r})\right|^{2} d \mathbf{r}}
\end{aligned}
$$

where $S_{1}$ is the region that is in the cylinder before displacement and now in the air, and $S_{2}$ is just the inverse. Note $S_{1}$ and $S_{2}$ have an equal size. Due to the continuous character of $\mathbf{E}_{0}(\mathbf{r})$ at the border of the cylinder, the field in $S_{1}$ and $S_{2}$ does not differ much at a weak disorder. Then the intergation of field in these two regions will cancel each other. Therefore, the frequency shift is small.

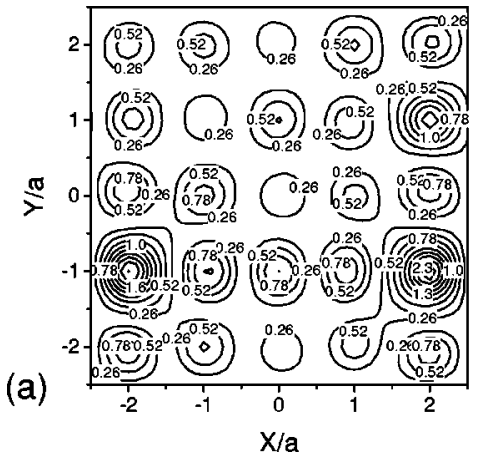

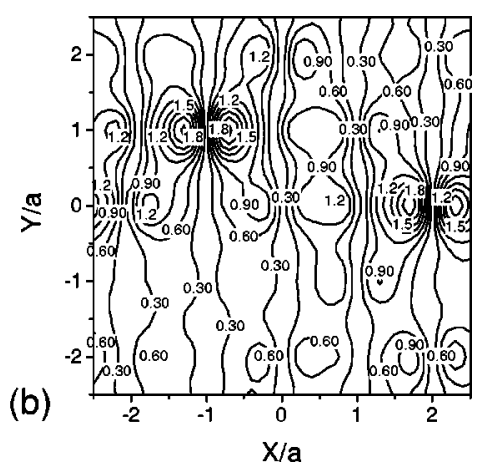

FIG. 12. The same as in Fig. 11, except that the random strength is $d_{r}=0.10$ and only the field distributions corresponding to the ground band gap are shown. 

is

The frequency shift when varying the radius of a cylinder

$$
\frac{\omega^{2}}{\omega_{0}^{2}}-1 \cong \frac{ \pm[\epsilon-1] \int_{S_{3}}\left|\mathbf{E}_{0}(\mathbf{r})\right|^{2} d \mathbf{r}}{\int_{S} \epsilon_{0}(\mathbf{r})\left|\mathbf{E}_{0}(\mathbf{r})\right|^{2} d \mathbf{r}},
$$

where $S_{3}$ is the region where the dielectric function changes, and " + ", and " - ", apply when the radius of the cylinder reduces and grows, respectively. As no cancellation effect occurs, it is clear that the frequency shift by such a size fluctuation is far more significant than in the case of a site randomness.

\section{SUMMARY AND CONCLUSIONS}

In summary, we have investigated photonic band gaps in disordered 2D photonic crystals of dielectric cylinders in air with two typical randomness of cylinder site displacement and cylinder radius variations. The DOS of disordered crystals are calculated by the plane-wave expansion method combined with a supercell technique to measure the band gap. To avoid powerful numerical calculations, a perturbation formalism is proposed to estimate band gaps in disordered crystals in a qualitative and semiquantitative way. The numerical simulations of DOS show that the band gap of the $E$-polarization mode is far more sensitive to disorders with a size variation than with a site displacement. The first and second band gaps both reduce very little for a site randomness with a strength as large as one-half the cylinder radius, yet they reduce more than one-half for a size randomness with a moderate strength of one-third the cylinder radius. Calculations on the frequency shift of edge states of band gaps with use of the approximate perturbation method accord well with the DOS results for disordered crystal with a site randomness of even a moderate strength. Yet the perturbation formalism is far less satisfactory in the case of a size randomness, especially for the higher band gaps. It only works well somewhat at a very weak strength of size randomness. The significant different behaviors of band-gap reduction by the site and size randomness can be understood by analyses of the electromagnetic fields in disordered crystals. It is found that the localization state appears far more easily in disordered crystal with even a weak size randomness than those with a strong site randomness. Such a strong localization effect reduces the band gaps significantly. The detailed field analysis also confirms the effectiveness of the proposed perturbation formalism. It is expected that such a simple perturbative analysis should provide a systematic way to understand various disordered photonic crystals qualitatively and even semiquantitatively.

\section{ACKNOWLEDGMENT}

The authors greatly acknowledge financial support from Hong Kong RGC Grant No. HKUST 6112/98P.
${ }^{1}$ See Photonic Band Gaps and Localization, Proceedings of the NATO ARW, edited by C. M. Soukoulis (Plenum, New York, 1993).

2 J. D. Joannopoulos, R. D. Meade, and J. N. Winn, Photonic Crystals (Princeton University Press, Princeton, 1995).

${ }^{3}$ E. Yablonovitch, Phys. Rev. Lett. 58, 2059 (1987).

${ }^{4}$ S. John, Phys. Rev. Lett. 58, 2486 (1987).

${ }^{5}$ S. John and J. Wang, Phys. Rev. Lett. 64, 2418 (1990); Phys. Rev. B 43, 12772 (1991).

${ }^{6}$ S. Y. Zhu, H. Chen, and H. Huang, Phys. Rev. Lett. 79, 205 (1997).

${ }^{7}$ J. D. Joannopoulos, P. R. Villeneuve, and S. Fan, Nature (London) (London) 386, 143 (1997).

${ }^{8}$ E. Yablonovitch, T. J. Gmitter, and K. M. Leung, Phys. Rev. Lett. 67, 2295 (1991).

${ }^{9}$ S. Y. Lin, J. G. Fleming, D. L. Hetherington, B. K. Smith, R. Biawas, K. M. Ho, M. M. Sigalas, W. Zurbrzycki, S. R. Kurtz, and J. Bur, Nature (London) (London) 394, 251 (1998).

${ }^{10}$ E. Özbay, E. Michel, G. Tuttel, R. Biswas, M. Sigalas, and K. M. Ho, Appl. Phys. Lett. 64, 2559 (1994).

${ }^{11}$ M. Wada, Y. Doi, K. Inoue, J. W. Haus, and Z. Yuan, Appl. Phys. Lett. 70, 2966 (1997).

${ }^{12}$ U. Grüning, V. Lehmann, S. Ottow, and K. Busch, Appl. Phys. Lett. 68, 747 (1996)

${ }^{13}$ T. Krauss, R. De La Rue, and S. Band, Nature (London) (London) 383, 699 (1996).

${ }^{14}$ K. Inoue, M. Wada, K. Sakoda, M. Hayashi, T. Fukushima, and A. Yamanaka, Phys. Rev. B 53, 1010 (1996).
${ }^{15}$ H. B. Lin, R. J. Tonucci, and A. J. Campillo, Appl. Phys. Lett. 68, 2927 (1996).

${ }^{16}$ R. D. Pradhan, J. A. Bloodgood, and G. H. Watson, Phys. Rev. B 55, 9503 (1997).

${ }^{17}$ I. I. Tarhan and G. H. Watson, Phys. Rev. Lett. 76, 315 (1996).

${ }^{18}$ S. Park and Y. Xia, Adv. Mater. 10, 1045 (1998); Z. Zhang, Y. Yin, B. Gates, and Y. Xia, ibid. 12, 206 (2000).

${ }^{19}$ Y. A. Vlasov, V. N. Astratov, O. Z. Karimov, A. A. Kaplyanskii, V. N. Bogomolov, and A. V. Prokofiev, Phys. Rev. B 55, R13 357 (1997).

${ }^{20}$ V. N. Bogolomov, S. V. Gaponenko, I. N. Germanenko, A. M. Kapitonov, E. P. Petrov, N. V. Gaponenko, A. V. Prokofiev, A. N. Ponyavina, N. I. Silvanovich, and S. M. Samoilovich, Phys. Rev. E 55, 7619 (1997).

${ }^{21}$ A. A. Zakhidov, R. H. Baughman, Z. Iqbal, C. C. Cui, I. Khayrullin, S. O. Dantas, J. Marti, and V. G. Ralchenko, Science 282, 897 (1998).

${ }^{22}$ J. E. G. J. Wijnhoven and W. L. Vos, Science 281, 802 (1998).

${ }^{23}$ S. Fan, P. R. Villeneuve, and J. D. Joannopoulos, J. Appl. Phys. 78, 1415 (1995)

${ }^{24}$ M. M. Sigalas, C. M. Soukoulis, C. T. Chan, and D. Turner, Phys. Rev. B 53, 8340 (1996).

${ }^{25}$ H. Q. Li, B. Y. Cheng, and D. Z. Zhang, Phys. Rev. B 56, 10734 (1997).

${ }^{26}$ A. Chutinan and S. Noda, J. Opt. Soc. Am. B 16, 240 (1999).

${ }^{27}$ M. M. Sigalas, C. M. Soukoulis, C. T. Chan, R. Biswas, and K. M. Ho, Phys. Rev. B 59, 12767 (1999). 
${ }^{28}$ H. Y. Ryu, J. K. Hwang, and Y. H. Lee, Phys. Rev. B 59, 5463 (1999).

${ }^{29}$ K. M. Ho, C. T. Chan, and C. M. Soukoulis, Phys. Rev. Lett. 65 , 3152 (1990).

${ }^{30}$ Z. Y. Li, J. Wang, and B. Y. Gu, Phys. Rev. B 58, 3721 (1998).
${ }^{31}$ Z. Y. Li, B. Y. Gu, and G. Z. Yang, Phys. Rev. Lett. 81, 2574 (1998).

${ }^{32}$ X. Zhang, Z. Q. Zhang, L. M. Li, C. J. Jin, D. Z. Zhang, B. Man, and B. Y. Cheng, Phys. Rev. B 61, 1892 (2000). 